\title{
High-Gain-Predictor-Based Output Feedback Control for Time-Delay Nonlinear Systems ${ }^{\circ}$
}

\author{
Jing Lei*, Hassan K. Khalil \\ This paper was not presented during the IFAC meetings \\ * Corresponding author. Email: elizabethia@126.com \\ Department of Electrical and Computer Engineering, Michigan State University, East Lansing, MI, 48824 USA, \\ Emails: elizabethia@126.com,khali1@msu.edu
}

\begin{abstract}
This paper designs a high-gain predictor for output feedback control of nonlinear systems in the presence of input, output, and state delays. The high-gain predictor realizes the states appearing in the output feedback control in terms of predictive state, delayed state, and current state. The system includes internal and external dynamics, and the closed-loop system under state feedback is required to be globally asymptotically stable and locally exponentially stable. Positively invariant sets are found to verify boundedness, exponential stability, and performance recovery. In the simulation, a saturated sliding mode control is applied to demonstrate the performance recovery of the closed-loop system, and the fact that the high-gainpredictor parameter has a lower bound related to time delays.
\end{abstract}

Key words: Nonlinear systems, time-delay systems, high-gain observers, output feedback control.

\section{Introduction}

In output feedback control of time-delay systems, state observers may be used to reconstruct the unmeasured states (e.g. Germani \& Pepe, 2005; Gemani, Manes, \& Pepe, 2012). But observer design for nonlinear time-delay systems is challenging. Gemani, Manes, \& Pepe (2002) applied the Gronwall lemma to provide sufficient conditions on delay for a chain observer to prove exponential convergence of the estimation error. These conditions were relaxed in Kazantzi \& Wright (2005). A Lyapunov-Krasovskii functional was introduced in Ahmed-Ali, Cherrier, \& M'Saad (2009) such that a relationship between the delay and the number of cascade observers with specific vector gains were proposed. Many authors employed this method in the later work (e.g. Farza, Sboui, Cherrier, \& M'Saad, 2010; Ghanes, Leon, \&Barbot, 2013; Ahmed-Ali, Van Assche, Massieu, \& Dorleans, 2013; Ahmed-Ali, Cherrier, \& Lamnabhi-Lagarrigue, 2012). The aformentioned papers are devoted to proving the exponential convergence of the observer estimation errors. Recently, Karagyllis, Krstic, Ahmed-Ali, \& Lamnabhi-Lagarrigue (2012) proved exponential stability of the closed-loop system in a disturbance free case. Those techniques for predictors (e.g., Gemani, Manes, \& Pepe, 2002; Karagyllis, Krstic, Ahmed-Ali, \& LamnabhiLagarrigue, 2012), work with time delays that are not necessarily small; however, they require exact models of the system. An advantage of high-gain observers is that we can dominate uncertain nonlinearities, while the price for that is restricting the delay to be small. Another advantage of using high-gain observers is that they can recover the performance of state feedback control.

In our previous work Lei and Khalil (2016), we used a high-gain predictor in feedback-linearization control. The system considered is nonlinear with time-varying input and output delays. To verify the performance recovery, we constructed a Lyapunov-Krasovskii functional, proved boundedness and exponential stability of the closed-loop system, and found a lower bound on the high-gain parameter, which relates to the maximum of involved delays. This work is an extension of Lei and Khalil (2016), which does the following: (1) we allow zero dynamics; (2) instead of feedback linearization, we allow any stabilizing state feedback control; (3) we allow state delay in addition to the input and output delays.

Notations. Throughout the paper, $|\cdot|$ means the absolute value; $I$ denotes the identity matrix; $\lambda$ denotes the eigenvalues, $\lambda_{\min }(\cdot)$ and $\lambda_{\max }(\cdot)$ are the minimum and maximum eigenvalues of the matrix respectively; the superscript of "T" stands for the transposition of a matrix; $R^{n}$ denotes the $n$-dimensional Euclidean space and $\|\cdot\|$ denotes the Euclidean norm; $R^{+}$represents the set of non-negative real numbers; given a positive constant $r \in R^{+}, L_{2}\left([-r, 0] ; R^{n}\right)$ denotes the space of square integrable functions $\varphi:[-r, 0] \rightarrow R^{n} ; \mathcal{C}^{n}$ denotes the space of absolutely continuous functions $\varphi:[-r, 0] \rightarrow R^{n}$, which have square inte- 
grable first-order derivatives; $x:[-r, \infty) \rightarrow R^{n}$ is a function; $x(t)$ means the value of $x$ at $t ; x_{t}$ is an element of $\mathcal{C}^{n}$ defined by $x_{t}(\theta)=x(t+\theta),-r \leq \theta \leq 0$ with the norm $\left\|x_{t}\right\|_{s}=\sup _{\theta \in[-r, 0]}\|x(t+\theta)\| \leq b$, where $b$ is a positive constant; $x\left(t ; t_{0}, \varphi\right)$ represents the solution of the given time-delay system with initial time $t_{0}$ and initial condition $\varphi$; given a Lyapunov functional $V\left(x_{t}, \dot{x}_{t}, t\right): \mathcal{C}^{n} \times L_{2} \times R \rightarrow R^{+}$, $\dot{V}\left(x_{t}, \dot{x}_{t}, t\right)=\lim _{h \rightarrow 0^{+}} \sup \frac{1}{h}\left[V\left(x_{t+h}, \dot{x}_{t+h}, t+h\right)-V\left(x_{t}, \dot{x}_{t}, t\right)\right] ;$ a scalar continuous function $\alpha(d)$, defined for $d \in[0, \bar{d})$, belongs to class $\mathcal{K}$ if it is strictly increasing and $\alpha(0)=0$.

\section{System description}

Consider a time-delay nonlinear system represented by

$$
\begin{aligned}
& \dot{\eta}(t)=\vartheta\left(\eta_{t}, \xi_{t}\right) \\
& \dot{\xi}(t)=A \xi(t)+B\left[\psi\left(\eta_{t}, \xi_{t}\right) u(t-\tau)+p\left(\eta_{t}, \xi_{t}\right)\right] \\
& y(t)=C \xi(t-s)
\end{aligned}
$$

where $\eta \in R^{t}, \xi \in R^{n-t}$ are the states, $u \in R$ is the control input, $y \in R$ is the output. The functions $\vartheta(\cdot), \psi(\cdot), p(\cdot)$ have multiple state delays, e.g.,

$$
\begin{array}{r}
\vartheta\left(\eta_{t}, \xi_{t}\right)=\vartheta\left(\eta(t), \eta\left(t-\bar{l}_{1}\right), \eta\left(t-\bar{l}_{2}\right), \cdots, \eta\left(t-\bar{l}_{m_{1}}\right) ;\right. \\
\left.\xi(t), \xi\left(t-l_{1}\right), \xi\left(t-l_{2}\right), \cdots, \xi\left(t-l_{m_{2}}\right)\right)
\end{array}
$$

where $\bar{l}_{j}\left(j=1,2, \cdots, m_{1}\right)$ and $l_{i}\left(i=1,2, \cdots, m_{2}\right)$ are state delays; $\psi(\cdot), p(\cdot)$ may have similar forms of state delays; $\tau$ and $s$ are known sufficiently small input and output delays respectively; and $\tau, s \in[0, r]$, where $r=\tau+s$. The initial conditions $\eta_{t_{0}}$ and $\xi_{t_{0}}$ are bounded and $\left\|\xi_{t_{0}}\right\|_{s} \leq \kappa_{1}$ with positive constant $\kappa_{1}$. The $(n-l) \times(n-\imath)$ matrix $A$, the $(n-\imath) \times 1$ matrix $B$, and the $1 \times(n-\imath)$ matrix $C$ represent a chain of $n-l$ integrators. System (1) is required to satisfy Assumption 1 below.

\section{Assumption 1.}

(i) The nonlinear functions $\vartheta(\cdot), \psi(\cdot), p(\cdot)$ are locally Lipschitz.

(ii) $\psi(\cdot) \neq 0$ and $1 / \psi(\cdot)$ is locally Lipschitz.

(iii) The origin $\left(\eta_{t}, \xi_{t}\right)=(0,0)$ is an equilibrium point of the unforced system; that is, $p(0,0)=0$ and $\vartheta(0,0)=0$.

Consider a partial state feedback control of the form $u(t)=\gamma\left(\xi(t+\tau), \xi\left(t+\tau-l_{1}\right), \xi\left(t+\tau-l_{2}\right), \cdots, \xi\left(t+\tau-l_{m_{2}}\right)\right)$ so that

$u(t-\tau)=\gamma\left(\xi(t), \xi\left(t-l_{1}\right), \xi\left(t-l_{2}\right), \cdots, \xi\left(t-l_{m_{2}}\right)\right) \triangleq \gamma\left(\xi_{t}\right)$ where the function $\gamma(\cdot)$ satisfies the following assumption.

\section{Assumption 2.}

(i) $\gamma(\cdot)$ is a locally Lipschitz function in $\xi_{t}$.

(ii) $\gamma(\cdot)$ is globally bounded in $\xi_{t}$.

(iii) The origin of the closed-loop system

$$
\begin{aligned}
& \dot{\eta}(t)=\vartheta\left(\eta_{t}, \xi_{t}\right) \\
& \dot{\xi}(t)=A \xi(t)+B\left[\psi\left(\eta_{t}, \xi_{t}\right) \gamma\left(\xi_{t}\right)+p\left(\eta_{t}, \xi_{t}\right)\right]
\end{aligned}
$$

$$
\text { where } \Xi \geq \max _{\xi \in \Omega_{c}}\left|\gamma_{0}\left(\xi_{t}\right)\right| \text {. Therefore, for every } \xi(0) \in \Omega_{c} \text {, }
$$
$\left|\gamma\left(\xi_{t}\right)\right| \leq \Xi$. Now the function $\gamma\left(\xi_{t}\right)$ satisfies all the conditions of Assumption 2.

\section{High-gain predictor design}

We will use $\hat{\xi}(t), \hat{\xi}\left(t-l_{1}\right), \hat{\xi}\left(t-l_{2}\right), \ldots, \hat{\xi}\left(t-l_{m_{2}}\right)$ in the output feedback control in order to replace the states $\xi(t+\tau), \xi\left(t+\tau-l_{1}\right), \xi\left(t+\tau-l_{2}\right), \ldots, \xi\left(t+\tau-l_{m_{2}}\right)$ in the state feedback (3). We now construct a high-gain predictor to generate $\hat{\xi}(t)$ and delay it in the observer equation according to the state delays.

The high-gain predictor takes the form 


$$
\begin{aligned}
& \dot{\hat{\xi}}(t)= A \hat{\xi}(t)+B\left[\psi_{0}\left(\hat{\xi}_{t}\right) u(t)+p_{0}\left(\hat{\xi}_{t}\right)\right] \\
&+H[y(t)-C \hat{\xi}(t-s-\tau)]
\end{aligned}
$$

with the initial condition $\hat{\xi}_{t_{0}}$ satisfying $\left\|\hat{\xi}_{t_{0}}\right\|_{s} \leq \hat{\kappa}_{1}$, where $\hat{\kappa}_{1}$ is a positive constant. The functions $\psi_{0}$ and $p_{0}$ are nominal models of $\psi$ and $p$; they are bounded functions of their arguments. The observer gain $H$ is given by $H=\left[a_{1} / \varepsilon, a_{2} / \varepsilon^{2}, \cdots, a_{n-l} / \varepsilon^{n-l}\right]^{\mathrm{T}}$, in which $\varepsilon$ is a small positive constant to be specified and $a_{j}(j=1,2, \cdots, n-\imath)$ are positive constants chosen such that all the roots of the characteristic equation $\lambda^{n-l}+a_{1} \lambda^{n-l-1}+\cdots+a_{n-l-1} \lambda+a_{n-l}=0$ have negative real parts.

The state $\hat{\xi}(t)$ of the predictor is an estimate of $\xi(t+\tau)$. The estimation error $e(t)=\xi(t)-\hat{\xi}(t-\tau)$ satisfies the equation

$$
\begin{aligned}
\dot{e}(t)= & A e(t)-H C e(t-s-\tau)+B\left\{\left[\psi\left(\eta_{t}, \xi_{t}\right)-\psi_{0}\left(\hat{\xi}_{t-\tau}\right)\right] u(t-\tau)\right. \\
& \left.+p\left(\eta_{t}, \xi_{t}\right)-p_{0}\left(\hat{\xi}_{t-\tau}\right)\right\}
\end{aligned}
$$

For the purpose of analysis, we replace the error system (8) by the equivalent dynamics of the scaled estimation error $\varsigma(t)=N^{-1}(\varepsilon) e(t)$, where $N(\varepsilon)=\operatorname{diag}\left(\varepsilon^{n-l-1}, \quad \varepsilon^{n-l-2}, \cdots, \varepsilon, 1\right)$. Then, the scaled error system is described by

$$
\begin{aligned}
\varepsilon \dot{\zeta}(t)= & A \varsigma(t)-B_{0} C \varsigma(t-s-\tau)+\varepsilon B\left\{\left[\psi\left(\eta_{t}, \xi_{t}\right)-\psi_{0}\left(\hat{\xi}_{t-\tau}\right)\right]\right. \\
& \left.\times u(t-\tau)+p\left(\eta_{t}, \xi_{t}\right)-p_{0}\left(\hat{\xi}_{t-\tau}\right)\right\}
\end{aligned}
$$

where $\quad B_{0}=\left[a_{1}, a_{2}, \cdots, a_{n-l}\right]^{\mathrm{T}} \quad, \quad A=\varepsilon N^{-1}(\varepsilon) A N(\varepsilon)$ $B_{0} C=\varepsilon N^{-1}(\varepsilon) H C N(\varepsilon)$, and $B=N^{-1}(\varepsilon) B$.

Applying the bounded output feedback control

$$
u(t)=\gamma\left(\hat{\xi}(t), \hat{\xi}\left(t-l_{1}\right), \hat{\xi}\left(t-l_{2}\right), \cdots, \hat{\xi}\left(t-l_{m_{2}}\right)\right) \triangleq\left(\hat{\xi}_{t}\right)
$$

to system (1), the closed-loop system is

$$
\begin{aligned}
\dot{\eta}(t)= & \vartheta\left(\eta_{t}, \xi_{t}\right) \\
\dot{\xi}(t)= & A \xi(t)+B\left[\psi ( \eta _ { t } , \xi _ { t } ) \gamma \left(\hat{\xi}(t-\tau), \hat{\xi}\left(t-\tau-l_{1}\right),\right.\right. \\
& \left.\left.\hat{\xi}\left(t-\tau-l_{2}\right), \cdots, \hat{\xi}\left(t-\tau-l_{m_{2}}\right)\right)+p\left(\eta_{t}, \xi_{t}\right)\right] \\
\varepsilon \dot{\zeta}(t)= & A \varsigma(t)-B_{0} C \varsigma(t-s-\tau)+\varepsilon B \delta\left(\eta_{t}, \xi_{t}, N(\varepsilon) \varsigma_{t}\right)
\end{aligned}
$$

where

$$
\begin{aligned}
\delta\left(\eta_{t}, \xi_{t}, e_{t}\right)= & {\left[\psi\left(\eta_{t}, \xi_{t}\right)-\psi_{0}\left(\hat{\xi}_{t-\tau}\right)\right] \gamma\left(\hat{\xi}_{t-\tau}\right) } \\
& +p\left(\eta_{t}, \xi_{t}\right)-p_{0}\left(\hat{\xi}_{t-\tau}\right)
\end{aligned}
$$

Due to Assumptions 1-2, $\delta\left(\eta_{t}, \xi_{t}, e_{t}\right)$ is locally Lipschitz in $\left(\eta_{t}, \xi_{t}, e_{t}\right)$. Moreover, $\delta(0,0,0)=0$.

We apply the transformation

$$
\varsigma(t-s-\tau)=\varsigma(t)-\int_{t-s-\tau}^{t} \dot{\zeta}(\varpi) \mathrm{d} \varpi
$$

into the third equation in (11), which results in

$$
\varepsilon \dot{\zeta}(t)=A_{0} \varsigma(t)+B_{0} C J+\varepsilon B \delta\left(\eta_{t}, \xi_{t}, N(\varepsilon) \varsigma_{t}\right)
$$

where $A_{0}=A-B_{0} C$ is Hurwitz and $J=\int_{t-s-\tau}^{t} \dot{\zeta}(\varpi) \mathrm{d} \varpi$. Thus, we rewrite the closed-loop system (11) in a compact singularly perturbed form:

$$
\begin{gathered}
\dot{\chi}(t)=F\left(\chi_{t}, N(\varepsilon) \varsigma_{t}\right) \\
\varepsilon \dot{\zeta}(t)=A_{0} \varsigma(t)+B_{0} C J+\varepsilon B \delta\left(\chi_{t}, N(\varepsilon) \varsigma_{t}\right)
\end{gathered}
$$

with the initial conditions $\chi_{t_{0}}=\left[\eta_{t_{0}}^{\mathrm{T}}, \xi_{t_{0}}^{\mathrm{T}}\right]^{\mathrm{T}} \in \Omega_{c_{0}}$ and $\hat{\xi}_{t_{0}} \in \mathcal{Q}$, where $\Omega_{c_{0}}=\left\{V_{1}\left(\chi_{t}\right) \leq c_{0}\right\}, c_{0}$ is a positive constant, and $\mathcal{Q}$ are any compact subsets of $\mathcal{A}$ and $\mathcal{C}^{n-l}$ respectively. Both the states $\chi$ and $\varsigma$ in system (15) are of time delay, which requires significant changes in the stability analysis comparing with those without delay.

\section{Performance recovery}

In this section, we will show that the closed-loop system (15) recovers the performance of the closed-loop system under state feedback (4), which is the reduced system of (15). Before it, we do the following preliminary.

\subsection{Unperturbed systems}

Setting $\varepsilon=0$ in $(15-2)$ yields $\varsigma_{t}=0$ and the reduced system

$$
\dot{\chi}=F\left(\chi_{t}, 0\right)
$$

which is the closed-loop system under the state feedback control (4). By Assumption 2, the origin of (16) is asymptotically stable with a region of attraction and locally exponentially stable.

For the unperturbed fast system

$$
\varepsilon \dot{\zeta}(t)=A \varsigma(t)-B_{0} C \varsigma(t-s-\tau)=A_{0} \varsigma(t)+B_{0} C J
$$

define a Lyapunov-Krasovskii functional candidate:

$$
V_{3}\left(\varsigma_{t}, \dot{\zeta}_{t}\right)=\varsigma^{\mathrm{T}}(t) P \varsigma(t)+\int_{t-r}^{t}(\varpi-t+r)\|\dot{\zeta}(\varpi)\|^{2} \mathrm{~d} \varpi
$$

where $V_{3}\left(\varsigma_{t}, \dot{\zeta}_{t}\right): \mathcal{C}^{n-t} \times L_{2} \rightarrow R^{+}, P$ is the symmetric positive definite solution of the algebraic Lyapunov equation $A_{0}^{\mathrm{T}} P+P A_{0}=-I$. We will prove that (17) is globally exponentially stable. The derivative $\dot{V}_{3}\left(\varsigma_{t}, \dot{\zeta}_{t}\right)$ along the system (17) satisfies

$$
\begin{aligned}
\dot{V}_{3} \leq & -\frac{1}{\varepsilon}\|\varsigma(t)\|^{2}+\frac{2}{\varepsilon}\left\|P B_{0} C\right\|\|\varsigma(t)\|\|J\|+r\|\dot{\zeta}(t)\|^{2} \\
& -\int_{t-r}^{t}\|\dot{\zeta}(\varpi)\|^{2} \mathrm{~d} \varpi
\end{aligned}
$$

Using Young's inequality, from the second equation in (17),

$$
\|\dot{\zeta}(t)\|^{2} \leq \frac{2\left\|A_{0}\right\|^{2}}{\varepsilon^{2}}\|\varsigma(t)\|^{2}+\frac{2\left\|B_{0} C\right\|^{2}}{\varepsilon^{2}}\|J\|^{2}
$$

Also, the following inequality holds:

$$
\begin{aligned}
& -\frac{1}{2 \varepsilon}\|\varsigma(t)\|^{2}+\frac{2}{\varepsilon}\left\|P B_{0} C\right\|\|\varsigma(t)\|\|J\| \\
= & -\frac{1}{2 \varepsilon}\left[\left(\|\varsigma(t)\|-2\left\|P B_{0} C\right\|\|J\|\right)^{2}-4\left\|P B_{0} C\right\|^{2}\|J\|^{2}\right] \\
\leq & \frac{2\left\|P B_{0} C\right\|^{2}}{\varepsilon}\|J\|^{2}
\end{aligned}
$$

Applying Jensen's inequality $\|J\|^{2} \leq r \int_{t-r}^{t}\|\dot{\zeta}(\varpi)\|^{2} \mathrm{~d} \varpi \quad$ and substituting (20), (21) into (19) yields 


$$
\begin{aligned}
\dot{V}_{3} \leq & -\frac{1}{4 \varepsilon}\|\zeta(t)\|^{2}-\left[\frac{1}{4 \varepsilon}-\frac{2 r\left\|A_{0}\right\|^{2}}{\varepsilon^{2}}\right]\|\varsigma(t)\|^{2} \\
& -\left[1-\frac{2\left\|P B_{0} C\right\|^{2} r}{\varepsilon}-\frac{2\left\|B_{0} C\right\|^{2} r^{2}}{\varepsilon^{2}}\right] \int_{t-r}^{t}\|\dot{\zeta}(\varpi)\|^{2} \mathrm{~d} \varpi \\
\leq- & \frac{1}{4 \varepsilon}\|\zeta(t)\|^{2}-\frac{1}{2 r} r \int_{t-r}^{t}\|\dot{\zeta}(\varpi)\|^{2} \mathrm{~d} \sigma-\left[\frac{1}{4 \varepsilon}-\frac{2 r\left\|A_{0}\right\|^{2}}{\varepsilon^{2}}\right]\|\zeta(t)\|^{2} \\
& -\left[\frac{1}{2}-\frac{2\left\|P B_{0} C\right\|^{2} r}{\varepsilon}-\frac{2\left\|B_{0} C\right\|^{2} r^{2}}{\varepsilon^{2}}\right] \int_{t-r}^{t}\|\dot{\zeta}(\varpi)\|^{2} \mathrm{~d} \varpi
\end{aligned}
$$

Then, taking

where

$$
\varepsilon \geq \sigma r
$$

$$
\sigma=\max \left\{8\left\|A_{0}\right\|^{2}, 2\left\|B_{0} C\right\|, 4\left\|P B_{0} C\right\|^{2}\right\}
$$

the coefficients of the last two terms in (22) are nonnegative. Since

$$
r \int_{t-r}^{t}\|\dot{\zeta}(\varpi)\|^{2} \mathrm{~d} \sigma \geq \int_{t-r}^{t}(\sigma-t+r)\|\dot{\mathcal{S}}(\varpi)\|^{2} \mathrm{~d} \sigma
$$

inequality (22) becomes

$$
\begin{aligned}
\dot{V}_{3} \leq & -\frac{1}{4 \varepsilon}\|\varsigma(t)\|^{2}-\frac{1}{2 r} \int_{t-r}^{t}(\varpi-t+r)\|\dot{\zeta}(\varpi)\|^{2} \mathrm{~d} \varpi \\
\leq & -\frac{1}{\varepsilon}\left[\frac{\varsigma^{\mathrm{T}}(t) P \zeta(t)}{4\|P\|}+\frac{\sigma}{2} \int_{t-r}^{t}(\varpi-t+r)\|\dot{\zeta}(\varpi)\|^{2} \mathrm{~d} \varpi\right] \\
\leq & -\frac{1}{\varepsilon} \min \left\{\frac{1}{4\|P\|}, \frac{\sigma}{2}\right\}\left[\varsigma^{\mathrm{T}}(t) P_{\zeta}(t)\right. \\
& \left.+\int_{t-r}^{t}(\varpi-t+r)\|\dot{\zeta}(\varpi)\|^{2} \mathrm{~d} \varpi\right] \\
\triangleq & -\frac{\tilde{c}_{3}}{\varepsilon} V_{3}
\end{aligned}
$$

where $\tilde{c}_{3}=\min \left\{\frac{1}{4\|P\|}, \frac{\sigma}{2}\right\}$. Moreover, from the first equation in (17),

$$
\|\dot{\zeta}(t)\|^{2} \leq \frac{2}{\varepsilon^{2}}\left[\|A\|^{2}+\left\|B_{0} C\right\|^{2}\right]\left\|S_{t}\right\|_{s}^{2}
$$

Thus, from (18),

where

$$
\tilde{c}_{1}\|S\|^{2} \leq V_{3} \leq \tilde{c}_{2}\left\|s_{t}\right\|_{s}^{2}
$$

$$
\tilde{c}_{1}=\lambda_{\min }(P), \quad \tilde{c}_{2}=\lambda_{\max }(P)+\frac{2 r^{2}}{\varepsilon^{2}}\left[\|A\|^{2}+\left\|B_{0} C\right\|^{2}\right]
$$

From (25) and (27), according to comparison principle (Khalil, 2002),

$$
\tilde{c}_{1}\|S(t)\|^{2} \leq V_{3}\left(\varsigma_{t}, \dot{S}_{t}\right) \leq V_{3}\left(\varsigma_{t_{0}}, \dot{\zeta}_{t_{0}}\right) \mathrm{e}^{-\frac{\tilde{c}_{\frac{3}{3}}}{\varepsilon}\left(t-t_{0}\right)} \leq \tilde{c}_{2}\left\|S_{t_{0}}\right\|_{s}^{2} \mathrm{e}^{-\frac{\tilde{c}_{3}}{\varepsilon}\left(t-t_{0}\right)}
$$

which deduces

$$
\|S(t)\| \leq \sqrt{\frac{\tilde{c}_{2}}{\tilde{c}_{1}}}\left\|S_{t_{0}}\right\|_{s} \mathrm{e}^{-\frac{\tilde{s}_{3}}{2 \varepsilon}\left(t-t_{0}\right)}
$$

From the definition of exponentially stability of time-delay systems (Krasovskii, 1963), system (17) is globally exponentially stable.
Remark 2. In the condition (23), $r$ is assumed sufficiently small so that $\varepsilon$ can be chosen to satisfy the condition, which implies that the input and output time delays are sufficiently small.

To study the perturbed fast system (15-2), we need a Lyapunov function which is bounded from below by a term proportional to $\left\|s_{t}\right\|_{s}^{2}$. The functional (18), which we used to study the unperturbed system (17), does not have this property because it is bounded from below by $\tilde{c}_{1}\left\|\varsigma_{\varsigma}\right\|^{2}$. Therefore, we use a converse Lyapunov functional $V_{4}\left(\varsigma_{t}, t, \varepsilon\right)$ for (17) that satisfies

$$
\begin{gathered}
\bar{c}_{1}\left\|\varsigma_{t}\right\|_{s}^{2} \leq V_{4} \leq \bar{c}_{2}\left\|s_{t}\right\|_{s}^{2}, \quad \dot{V}_{4} \leq-\frac{\bar{c}_{3}}{\varepsilon}\left\|\varsigma_{t}\right\|_{s}^{2} \\
\left|V_{4}\left(\varsigma_{t}^{\prime \prime}, t, \varepsilon\right)-V_{4}\left(\varsigma_{t}^{\prime}, t, \varepsilon\right)\right| \leq \bar{c}_{4}\left(\left\|s_{t}^{\prime \prime}\right\|+\left\|s_{s}^{\prime}\right\|_{s}\right)\left\|\varsigma_{t}^{\prime \prime}-\varsigma_{t}^{\prime}\right\|_{s}
\end{gathered}
$$

with some positive constants $\bar{c}_{1}, \bar{c}_{2}, \bar{c}_{3}, \bar{c}_{4}$, such a functional follows from a straight forward modification of the proof of Lemma 33.1 of Krasovskii (1963).The proof is concluded in Lemma 1 of the appendix.

\subsection{Main results}

Performance recovery of the closed-loop system under the output feedback control (10), with sufficiently small $\varepsilon$, shows in three aspects: first, the origin of the closed-loop system (15) under the output feedback control is exponentially stable; second, under the output feedback control the set of initial conditions $\Omega_{c_{0}} \times \mathcal{Q}$ can be chosen to include any compact subset of $\mathcal{A}^{\circ} \times \mathcal{C}^{n-t}$ and when $\mathcal{A}=\mathcal{C}^{n}$, the control achieves semiglobal stabilization; third, the trajectory under output feedback approaches that under state feedback as $\varepsilon$ decreases.

Choose a positive constant $c$ such that $c>c_{0}$. Then, $\Omega_{c_{0}} \subset \Omega_{c} \subset \mathcal{A}$. Denote the sets $\Sigma=\left\{V_{4} \leq \varepsilon^{2} \rho\right\} \subset \mathcal{C}^{n-t}$ and $\Lambda=\Omega_{c} \times \Sigma \subset \mathcal{A} \times \mathcal{C}^{n-l}$, where $\rho$ is a positive constant.

Theorem 1. Consider the closed-loop system (15) of the plant (1) and the output feedback controller (10). Let Assumptions1-2 hold, $\Omega_{c_{0}}$ be any compact set in the interior of $\mathcal{A}$, and $\mathcal{Q}$ be any compact subset of $\mathcal{C}^{n-t}$. Then, there exist $\sigma>0, \varepsilon_{1}^{*}>\sigma r$, and $T(\varepsilon)>t_{0}$ with $\lim T(\varepsilon)=0$, where $r=\tau+s$, such that for every $r<\varepsilon_{1}^{*} / \sigma$ and $\sigma r \leq \varepsilon \leq \varepsilon_{1}^{*}$, the trajectory of the closed-loop system, starting in $\Omega_{c_{0}} \times \mathcal{Q}$, is bounded for all $t \geq t_{0}$ and enters $\Lambda$ within the time interval $t \in\left[t_{0}, T(\varepsilon)\right]$ and remain thereafter.

Proof. We will show that there exist positive constants $\sigma$, $\rho$, and $\varepsilon_{1}$ (dependent on $\rho$ ) such that the compact set $\Lambda$ is positively invariant for every $\sigma r \leq \varepsilon \leq \varepsilon_{1}$. This will be done by showing that $\dot{V}_{1} \leq 0$ for $\left(\chi_{t}, \varsigma_{t}\right) \in\left\{V_{1}=c\right\} \times \Sigma$ and $\dot{V}_{4} \leq 0$ for all $\left(\chi_{t}, \varsigma_{t}\right) \in \Omega_{c} \times\left\{V_{4}=\varepsilon^{2} \rho\right\}$.

For an arbitrary $\tilde{\varepsilon}<1$, there exists $k_{1}$, which is independent of $\varepsilon$, such that for all $\chi_{t} \in \Omega_{c}$ and every $\varepsilon \leq \tilde{\varepsilon}$,

$$
\left\|F\left(\chi_{t}, N(\varepsilon) \varsigma_{t}\right)-F\left(\chi_{t}, 0\right)\right\|_{s} \leq k_{1}\left\|_{s_{t}}\right\|_{s}
$$

Due to the properties in (5) and (29), the derivative of $V_{1}\left(\chi_{t}\right)$ along the closed-loop system (15) satisfies 


$$
\begin{aligned}
\dot{V}_{1}= & \lim _{h \rightarrow 0^{+}} \sup \frac{1}{h}\left[V_{1}\left(F\left(\chi_{t}, 0\right) h+\chi_{t}\right)-V_{1}\left(\chi_{t}\right)\right]+\lim _{h \rightarrow 0^{+}} \sup \frac{1}{h} \\
& \times\left[V_{1}\left(F\left(\chi_{t}, N(\varepsilon) S_{t}\right) h+\chi_{t}\right)-V_{1}\left(F\left(\chi_{t}, 0\right) h+\chi_{t}\right)\right] \\
\leq & -\alpha_{3}\left(\left\|\chi_{t}\right\|_{s}\right)+L_{1} k_{1}\left\|s_{t}\right\|_{s}
\end{aligned}
$$

where $L_{1}$ is the Lipschitz constant of $V_{1}$. From (28),

$$
\left\|\varsigma_{t}\right\|_{s} \leq \sqrt{\frac{V_{4}}{\bar{c}_{1}}} \leq \varepsilon \sqrt{\frac{\rho}{\bar{c}_{1}}}
$$

Hence, when

$$
\varepsilon \leq \frac{\beta}{k_{1} L_{1}} \sqrt{\frac{\overline{c_{1}}}{\rho} \triangleq} \bar{\varepsilon}_{1}
$$

$\dot{V}_{1} \leq 0$, where $\beta=\min _{V_{1}=c} \alpha_{3}\left(\left\|\chi_{t}\right\|_{s}\right)$. fies

The derivative $\dot{V}_{4}\left(\varsigma_{t}, t, \varepsilon\right)$ along the system (15-2) satis-

$$
\begin{aligned}
\dot{V}_{4} & =\lim _{h \rightarrow 0^{+}} \sup \frac{1}{h}\left[V_{4}\left(\varsigma_{t+h}, t+h, \varepsilon\right)-V_{4}\left(\varsigma_{t}, t, \varepsilon\right)\right] \\
& =\left.\dot{V}_{4}\right|_{(17)}+\lim _{h \rightarrow 0^{+}} \sup \frac{1}{h}\left[V_{4}\left(\varsigma_{t+h}, t+h, \varepsilon\right)-V_{4}\left(\varsigma_{t+h}^{(u)}, t+h, \varepsilon\right)\right]
\end{aligned}
$$

where $\varsigma_{t+h}^{(u)}$ is the solution of (17) at $t+h$ starting from $\varsigma_{t}$ at $t$. Thus with the properties of (28), there is $k_{2}>0$ such that

$$
\dot{V}_{4} \leq-\frac{\bar{c}_{3}}{\varepsilon}\left\|\varsigma_{t}\right\|_{s}^{2}+k_{2}\left\|s_{t}\right\|_{s}\|\delta\|
$$

Because $\Omega_{c}$ is compact, $\psi, \psi_{0}, p, p_{0}$ are locally Lipschitz, and $\gamma\left(\hat{\xi}_{t}\right)$ is bounded, the uncertainty $\delta$ has the property:

$$
\|\delta\| \leq \beta_{1}\left\|_{S_{t}}\right\|_{s}+\beta_{2}
$$

where $\beta_{1}, \beta_{2}$ are positive constants independent of $\varepsilon$. Consequently,

$$
\dot{V}_{4}+\frac{\sigma_{1}}{\varepsilon} V_{4} \leq-\left[\frac{\bar{c}_{3}}{\varepsilon}-k_{2} \beta_{1}-\frac{\sigma_{1} \bar{c}_{2}}{\varepsilon}\right]\left\|s_{t}\right\|_{s}^{2}+k_{2} \beta_{2}\left\|\varsigma_{t}\right\|_{s}
$$

Taking $\sigma_{1} \leq \frac{\bar{c}_{3}}{4 \bar{c}_{2}}$ and $\varepsilon<\frac{\bar{c}_{3}}{4 k_{2} \beta_{1}} \triangleq \bar{\varepsilon}_{2}$,

$$
\dot{V}_{4}+\frac{\sigma_{1}}{\varepsilon} V_{4} \leq-\frac{\bar{c}_{3}}{2 \varepsilon}\left\|\varsigma_{t}\right\|_{s}^{2}+k_{2} \beta_{2}\left\|\varsigma_{t}\right\|_{s}
$$

Defining

$$
\rho=\frac{4 \bar{c}_{1} k_{2}^{2} \beta_{2}^{2}}{\bar{c}_{3}^{2}}
$$

then, from (37), when $V_{4} \geq \varepsilon^{2} \rho$,

$$
\dot{V}_{4} \leq-\frac{\sigma_{1}}{\varepsilon} V_{4}
$$

Therefore, for every $\sigma r \leq \varepsilon \leq \varepsilon_{1}$ with $\varepsilon_{1}=\min \left\{\bar{\varepsilon}_{1}, \bar{\varepsilon}_{2}\right\}$, we have $\dot{V}_{1} \leq 0$ for all $\left(\chi_{t}, s_{t}\right) \in\left\{V_{1}=c\right\} \times \Sigma$ and $\dot{V}_{4} \leq 0$ for all $\left(\chi_{t}, \varsigma_{t}\right) \in \Omega_{c} \times\left\{V_{4}=\varepsilon^{2} \rho\right\}$. Hence, $\Lambda$ is positively invariant.

Now, we will prove that the trajectory of the closedloop system is bounded. Since for every $\chi_{t} \in \Omega_{c}, F$ is globally bounded in $\hat{\xi}$,

$$
\left\|F\left(\chi_{t}, N(\varepsilon) \varsigma_{t}\right)\right\|_{s} \leq k
$$

where $k$ is a positive constant independent of $\varepsilon$. Consider the initial state $\left(\chi_{t_{0}}, \varsigma_{t_{0}}\right) \in \Omega_{c_{0}} \times \mathcal{Q}$. Since $\chi_{t_{0}}$ is in the interior of $\Omega_{c}$, it can be shown that

$$
\left\|\chi(t)-\chi\left(t_{0}\right)\right\| \leq \int_{t_{0}}^{t}\left\|F\left(\chi_{\pi}, N(\varepsilon) \zeta_{\varpi}\right)\right\|_{s} \mathrm{~d} \varpi \leq k\left(t-t_{0}\right)
$$

as long as $\chi_{t} \in \Omega_{c}$. Thus, there exists a finite time $T_{1}$ independent of $\varepsilon$ such that for every $t \in\left[t_{0}, T_{1}\right], \chi_{t} \in \Omega_{c}$. During this time interval, it follows from (39) that

$$
V_{4}\left(\varsigma_{t}, t, \varepsilon\right) \leq V_{4}\left(\varsigma_{t_{0}}, t_{0}, \varepsilon\right) \mathrm{e}^{-\frac{\sigma_{1}}{\varepsilon}\left(t-t_{0}\right)}
$$

Due to the initial condition,

$$
\left\|\varsigma_{t_{0}}\right\|_{s} \leq\left\|N^{-1}(\varepsilon)\right\|\left\|\xi_{t_{0}}-\hat{\xi}_{t_{0}}\right\|_{s} \leq \frac{\left\|\xi_{t_{0}}\right\|_{s}+\left\|\hat{\xi}_{t_{0}}\right\|_{s}}{\varepsilon^{n-1}} \leq \frac{\kappa_{1}+\hat{\kappa}_{1}}{\varepsilon^{n-1}}
$$

and $V_{4}\left(\varsigma_{t_{0}}, t_{0}, \varepsilon\right) \leq \bar{c}_{2}\left\|\varsigma_{t_{0}}\right\|_{s}^{2}$, hence,

$$
V_{4}\left(\varsigma_{t_{0}}, t_{0}, \varepsilon\right) \leq \frac{\bar{c}_{2}\left(\kappa_{1}+\hat{\kappa}_{1}\right)^{2}}{\varepsilon^{2(n-1)}} \triangleq \frac{\kappa}{\varepsilon^{2(n-1)}}
$$

Thus, the decay rate is obtained as

$$
V_{4}(\varsigma, t, \varepsilon) \leq \frac{\kappa}{\varepsilon^{2(n-1)}} \mathrm{e}^{-\frac{\sigma_{1}}{\varepsilon}\left(t-t_{0}\right)}
$$

which indicates that within the finite time $T(\varepsilon)=\frac{\varepsilon}{\sigma_{1}} \ln \left[\frac{\kappa}{\rho \rho^{2 n}}\right]+t_{0}$, the trajectory enters the set $\left\{V_{4} \leq \varepsilon^{2} \rho\right\}$; that is, for all $t \geq T(\varepsilon), V_{4} \leq \varepsilon^{2} \rho$. Hence, $\|\varsigma(t)\| \leq \varepsilon \sqrt{\rho / \bar{c}_{1}}=O(\varepsilon)$.

Thereby, we can choose $\varepsilon_{2}>\sigma r$ small enough so that $T(\varepsilon) \leq \frac{1}{2} T_{1}$ for all $\sigma r \leq \varepsilon \leq \varepsilon_{2}$. Note that $\varepsilon_{2}$ exists, because $T(\varepsilon)$ tends to zero as $\varepsilon$ tends to zero and $r$ is required to be sufficiently small. It follows that $V_{4}\left(\varsigma_{T(\varepsilon)}, T(\varepsilon), \varepsilon\right) \leq \varepsilon^{2} \rho$ for every $\sigma r \leq \varepsilon \leq \varepsilon_{2}$. Then, taking $\varepsilon_{1}^{*}=\min \left\{\tilde{\varepsilon}, \varepsilon_{1}, \varepsilon_{2}\right\}$ guarantees that, for every $\sigma r \leq \varepsilon \leq \varepsilon_{1}^{*}$, the trajectory will enter $\Lambda$ during the time interval $t \in\left[t_{0}, T(\varepsilon)\right]$ and remain there for $t \geq T(\varepsilon)$. Hence, the trajectory is bounded for all $t \geq T(\varepsilon)$. In addition, for $t \in\left[t_{0}, T(\varepsilon)\right]$, the trajectory is bounded by virtue of inequalities (41) and (42). Therefore, the trajectory is bounded for all $t \geq t_{0}$. Thus, the boundedness proof is complete.

Theorem 2. Consider the closed-loop system (15) of the plant (1) and the output feedback controller (10). Let Assumptions1-2 hold, $\Omega_{c_{0}}$ be any compact set in the interior of $\mathcal{A}$, and $\mathcal{Q}$ be any compact subset of $\mathcal{C}^{n-t}$. Then, given any $v>0$, there exist $\sigma>0, \varepsilon_{2}^{*}>\sigma r$ and $T_{2}>t_{0}$ both dependent on $v$, where $r=\tau+s$, such that for every $r<\varepsilon_{2}^{*} / \sigma$ and $\sigma r \leq \varepsilon \leq \varepsilon_{2}^{*}$, the solution of the closed-loop system, starting in $\Omega_{c_{0}} \times \mathcal{Q}$, satisfies

$$
\|\chi(t)\| \leq v, \quad\|\hat{\xi}(t)\| \leq v, \quad \forall t \geq T_{2}
$$

Proof. Now, for every $\sigma r \leq \varepsilon \leq \varepsilon_{1}^{*}$, the solutions are inside the set $\Lambda$ for all $t \geq T(\varepsilon)$, where $\Lambda$ is $O(\varepsilon)$ in the direction of $\varsigma$. Thus, we can find $\varepsilon_{3}=\varepsilon_{3}(v) \leq \varepsilon_{1}^{*}$ such that for every $\sigma r \leq \varepsilon \leq \varepsilon_{3}$,

$$
\left\|s_{t}\right\|_{s} \leq \frac{1}{2} v, \quad \forall t \geq T\left(\varepsilon_{3}\right) \triangleq \bar{T}(v)
$$

From (30) and (31), $\dot{V}_{1} \leq-\alpha_{3}\left(\left\|\chi_{t}\right\|_{s}\right)+\varepsilon k_{1} L_{1} \sqrt{\rho / \bar{c}_{1}}$, for all $\left(\chi_{t}, \varsigma_{t}\right) \in \Lambda$; in other words, 


$$
\dot{V}_{1} \leq-\frac{1}{2} \alpha_{3}\left(\left\|\chi_{t}\right\|_{s}\right)
$$

for $\quad \chi_{t} \notin\left\{\alpha_{3}\left(\left\|\chi_{t}\right\|_{s}\right) \leq 2 \varepsilon k_{1} L_{1} \sqrt{\rho / \bar{c}_{1}} \triangleq \Phi(\varepsilon)\right\}$. For $\quad \alpha_{3}(\cdot) \quad$ is positive and continuous, the set $\left\{\alpha_{3}\left(\left\|\chi_{t}\right\|_{s}\right) \leq \Phi(\varepsilon)\right\}$ is compact for sufficiently small $\varepsilon$. Denote $\bar{c}(\varepsilon)=\max _{\alpha_{3}\left(\| \chi_{t_{t}}\right) \leq \Phi(\varepsilon)}\left\{V\left(\chi_{t}\right)\right\}$ where $\bar{c}(\varepsilon)$ is nondecreasing and $\lim _{\varepsilon \rightarrow 0} \bar{c}(\varepsilon)=0$, which means $\bar{c}$ can be made arbitrarily small by choosing $\varepsilon$ small enough. Considering the compact set $\Omega_{\bar{c}}=\left\{V\left(\chi_{t}\right) \leq \bar{c}(\varepsilon)\right\}$, we have $\left\{\alpha_{3}\left(\left\|\chi_{t}\right\|_{s}\right) \leq \Phi(\varepsilon)\right\} \subset \Omega_{\bar{c}}$. Choose $\varepsilon_{4}=\varepsilon_{4}(v) \leq \varepsilon_{1}^{*}$ small enough such that for all $\varepsilon \leq \varepsilon_{4},\left\{\alpha_{3}\left(\left\|\chi_{t}\right\|_{s}\right) \leq \Phi(\varepsilon)\right\}$ is compact, the set $\Omega_{\bar{c}}$ is in the interior of $\Omega_{c}$, and

$$
\Omega_{\bar{c}} \subset\left\{\left\|\chi_{t}\right\|_{s} \leq \frac{1}{2} v\right\}
$$

Then, for all $\chi_{t} \in \Omega_{c}$, but $\chi_{t} \notin \Omega_{\bar{c}}$, the inequality (48) holds. Therefore, $\Omega_{\bar{c}} \times \Sigma$ is positively invariant and every trajectory in $\Lambda$ will reach $\Omega_{\bar{c}} \times \Sigma$ in finite time, implying that given (49), there exists a finite time $\tilde{T}=\tilde{T}(v)$ such that for every $\sigma r \leq \varepsilon \leq \varepsilon_{4}$,

$$
\left\|\chi_{t}\right\|_{s} \leq \frac{1}{2} \nu, \quad \forall t \geq \tilde{T}
$$

Taking $\varepsilon_{2}^{*}=\varepsilon_{2}^{*}(v)=\min \left\{\varepsilon_{3}, \varepsilon_{4}\right\}$ and $T_{2}=T_{2}(v)=\max \{\bar{T}, \tilde{T}\}$, together with (47), (50), $\xi(t-\tau)=\xi(t)-N(\varepsilon) \xi(t)$, and $\|N(\varepsilon)\|=1,(46)$ is achieved. This completes the proof of ultimate boundedness.

Theorem 3. Consider the closed-loop system (15) of the plant (1) and the output feedback controller (10). Let Assumptions1-2 hold, $\Omega_{c_{0}}$ be any compact set in the interior of $\mathcal{A}$, and $\mathcal{Q}$ be any compact subset of $\mathcal{C}^{n-t}$. Then, there exist $\sigma>0$ and $\varepsilon_{3}^{*}>\sigma r$, where $r=\tau+s$, such that for every $r<\varepsilon_{3}^{*} / \sigma$ and $\sigma r \leq \varepsilon \leq \varepsilon_{3}^{*}$, the origin of the closedloop system is exponentially stable and $\Omega_{c_{0}} \times \mathcal{Q}$ is a subset of its region of attraction.

Proof. In the foregoing analysis, the trajectory of the closed-loop system enters the set $\Omega_{\bar{c}} \times \Sigma$ after the time $T_{2}$ and stays thereafter. Keep shrinking $\varepsilon$ to be small enough so that $\Omega_{\bar{c}} \subset \mathcal{B}_{b}$, meaning that there exists $\varepsilon_{5}>\sigma r$ such that for every $\sigma r \leq \varepsilon \leq \varepsilon_{5}$, the solution started from $\Omega_{c_{0}} \times \mathcal{Q}$ will enter $\mathcal{B}_{b} \times \Sigma$ in finite time.

Consider the composite Lyapunov functional candidate $V\left(\chi_{t}, \varsigma_{t}, t, \varepsilon\right)=V_{2}\left(\chi_{t}\right)+V_{4}\left(\varsigma_{t}, t, \varepsilon\right)$. We will study its derivative along the closed-loop system (15) for $\left(\chi_{t}, \varsigma_{t}\right) \in \mathcal{B}_{b} \times \Sigma$. Since inside the set $\mathcal{B}_{b} \times \Sigma, p_{0}$ is equivalent to the unbounded nominal function of $p$ and $p_{0}(0)=0$, the uncertainty $\delta$ has the property that

$$
\|\delta\| \leq \beta_{1}\left\|s_{t}\right\|_{s}+\beta_{2}\left\|\chi_{t}\right\|_{s}
$$

Thus, by replacing $\beta_{2}$ with $\beta_{2}\left\|\chi_{t}\right\|_{s}$ in the aforementioned derivations from (36) to (37), the derivative $\dot{V}_{4}$ along (152) becomes

$$
\dot{V}_{4}+\frac{\sigma_{1}}{\varepsilon} V_{4} \leq-\frac{\bar{c}_{3}}{2 \varepsilon}\left\|\varsigma_{t}\right\|_{s}^{2}+k_{2} \beta_{2}\left\|s_{t}\right\|_{s}\left\|\chi_{t}\right\|_{s}
$$

Hence, due to the properties of (6) with (52), the derivative $\dot{V}$ along (15) is

$$
\begin{aligned}
& \dot{V} \leq-c_{3}\left\|\chi_{t}\right\|_{s}^{2}+c_{4} \lim _{h \rightarrow 0^{+}} \sup \left[\left\|F\left(\chi_{t}, N(\varepsilon) \varsigma_{t}\right) h+\chi_{t}\right\|_{s}\right. \\
& \left.+\left\|F\left(\chi_{t}, 0\right) h+\chi_{t}\right\|_{s}\right]\left\|F\left(\chi_{t}, N(\varepsilon) s_{t}\right)-F\left(\chi_{t}, 0\right)\right\|_{s} \\
& -\frac{\sigma_{1}}{\varepsilon} V_{4}-\frac{\bar{c}_{3}}{2 \varepsilon}\left\|\varsigma_{t}\right\|_{s}^{2}+k_{2} \beta_{2}\left\|\varsigma_{t}\right\|_{s}\left\|\chi_{t}\right\|_{s} \\
& \leq-c_{3}\left\|\chi_{t}\right\|_{s}^{2}+\left(2 c_{4} k_{1}+k_{2} \beta_{2}\right)\left\|\chi_{t}\right\|_{s}\left\|s_{t}\right\|_{s}-\left[\frac{\bar{c}_{3}}{2 \varepsilon}+\frac{\sigma_{1} \bar{c}_{1}}{\varepsilon}\right]\left\|s_{t}\right\|_{s}^{2} \\
& =-\left[\left\|\chi_{t}\right\|_{s} \quad\left\|\varsigma_{t}\right\|_{s}\right] M\left[\begin{array}{l}
\left\|\chi_{t}\right\|_{s} \\
\left\|s_{t}\right\|_{s}
\end{array}\right] \\
& \leq-\lambda_{\min }(M)\left\|Z_{t}\right\|_{s}^{2}
\end{aligned}
$$

where

$$
\begin{gathered}
Z(t)=\left[\chi^{\mathrm{T}}(t), \varsigma^{\mathrm{T}}(t)\right]^{\mathrm{T}}, \quad Z_{t}=\left[\chi_{t}^{\mathrm{T}}, \varsigma_{t}^{\mathrm{T}}\right]^{\mathrm{T}} \\
\left\|Z_{t}\right\|_{s}=\sup _{\theta \in[-r, 0]}\left(\|\chi(t+\theta)\|^{2}+\|\varsigma(t+\theta)\|^{2}\right)^{1 / 2}=\left(\|\chi\|_{t}\left\|_{s}^{2}+\right\| \varsigma_{t} \|_{s}^{2}\right)^{1 / 2}
\end{gathered}
$$

and

$$
M=\left[\begin{array}{cc}
c_{3} & -c_{4} k_{1}-\frac{1}{2} k_{2} \beta_{2} \\
-c_{4} k_{1}-\frac{1}{2} k_{2} \beta_{2} & \frac{\bar{c}_{3}}{2 \varepsilon}+\frac{\sigma_{1} \bar{c}_{1}}{\varepsilon}
\end{array}\right]
$$

Choosing $\varepsilon<\frac{1}{2} c_{3}\left(\bar{c}_{3}+2 \sigma_{1} \bar{c}_{1}\right) /\left(c_{4} k_{1}+\frac{1}{2} k_{2} \beta_{2}\right)^{2} \triangleq \varepsilon_{6}$ ensures that the matrix $M$ is positive definite, which shows that $\lambda_{\min }(M)>0$. Actually, $\lambda_{\min }(M)$ is bounded from below by a positive constant that is independent of $\varepsilon$ (Luse \& Khalil, 1985). Moreover, $b_{1}\left\|Z_{t}\right\|_{s}^{2} \leq V \leq b_{2}\left\|Z_{t}\right\|_{s}^{2}$, where $b_{1}=\min \left\{c_{1}, \bar{c}_{1}\right\} \quad, \quad b_{2}=\max \left\{c_{2}, \bar{c}_{2}\right\} \quad$. Hence, $Z$ is exponentially stable in $\mathcal{B}_{b} \times \Sigma$ and there is $\varepsilon_{6}>\sigma r$ such that for every $\sigma r \leq \varepsilon \leq \varepsilon_{6}$, the origin is exponentially stable and every trajectory in $\mathcal{B}_{b} \times \Sigma$ converges to the origin as $t \rightarrow \infty$. Therefore, for every $\sigma r \leq \varepsilon \leq \varepsilon_{3}^{*}=\min \left\{\varepsilon_{5}, \varepsilon_{6}\right\}$, the origin is exponentially stable, and $\Omega_{c_{0}} \times \mathcal{Q}$ is a subset of the attraction region. The exponential stability proof is complete.

Theorem 4. Consider the closed-loop system (15) of the plant (1) and the output feedback controller (10). Let Assumptions1-2 hold, $\Omega_{c_{0}}$ be any compact set in the interior of $\mathcal{A}$, and $\mathcal{Q}$ be any compact subset of $\mathcal{C}^{n-t}$. Then, given any $v>0$, there exist $\sigma>0$ and $\varepsilon_{4}^{*}>\sigma r$ dependent on $v$, where $r=\tau+s$, such that for every $r<\varepsilon_{4}^{*} / \sigma$ and $\sigma r \leq \varepsilon \leq \varepsilon_{4}^{*}$, the solution of the closed-loop system, starting in $\Omega_{c_{0}} \times \mathcal{Q}$, satisfies

$$
\left\|\chi(t)-\chi_{r}(t)\right\| \leq v, \quad \forall t \geq t_{0}
$$

where $\chi_{r}$ is the solution of the reduced order system (16), starting at $\chi_{t_{0}}$.

Proof. Separate the time horizon $[0, \infty)$ in three parts: $[0, T(\varepsilon)],\left[T(\varepsilon), T_{2}\right]$, and $\left[T_{2}, \infty\right)$. Based on the foregoing analysis, the reduced order system (16) and the closed-loop system (15) are exponentially stability at the origin. Thus, there exists a finite time $T_{2} \geq T(\varepsilon)$ independent of $\varepsilon$ such 
that for every $\sigma r \leq \varepsilon \leq \varepsilon_{3}^{*}$,

$$
\left\|\chi(t)-\chi_{r}(t)\right\| \leq v, \quad \forall t \geq T_{2}
$$

Further, the inequality (41) shows that, when $t \in\left[t_{0}, T(\varepsilon)\right]$,

$$
\left\|\chi(t)-\chi\left(t_{0}\right)\right\| \leq k\left(t-t_{0}\right)
$$

By analogy,

$$
\left\|\chi_{r}(t)-\chi\left(t_{0}\right)\right\| \leq k\left(t-t_{0}\right), \quad \forall t \in\left[t_{0}, T(\varepsilon)\right]
$$

Thus, from (57) and (58),

$$
\left\|\chi(t)-\chi_{r}(t)\right\| \leq 2 k T(\varepsilon), \quad \forall t \in\left[t_{0}, T(\varepsilon)\right]
$$

Since $T(\varepsilon) \rightarrow 0$ as $\varepsilon \rightarrow 0$, there exists $\sigma r \leq \varepsilon_{3} \leq \varepsilon_{3}^{*}$ such that for every $\sigma r \leq \varepsilon_{3} \leq \varepsilon_{3}^{*}$,

$$
\left\|\chi(t)-\chi_{r}(t)\right\| \leq v, \quad \forall t \in\left[t_{0}, T(\varepsilon)\right]
$$

As for the interval $\left[T(\varepsilon), T_{2}\right]$, the solution $\chi(t)$ satisfies (15-1) with

$$
\left\|\chi(T(\varepsilon))-\chi_{r}(T(\varepsilon))\right\| \leq \Theta(\varepsilon)
$$

where $N(\varepsilon) \varsigma_{t}$ is $O(\varepsilon)$ and $\Theta(\varepsilon) \rightarrow 0$ as $\varepsilon \rightarrow 0$. Thus, there exists $\sigma r \leq \varepsilon_{4} \leq \varepsilon_{3}^{*}$ such that for every $\sigma r \leq \varepsilon_{4} \leq \varepsilon_{3}^{*}$,

$$
\left\|\chi(t)-\chi_{r}(t)\right\| \leq v, \quad \forall t \in\left[T(\varepsilon), T_{2}\right]
$$

(Th. 1.3, Kharitonov, 2012). Take $\varepsilon_{3}^{*}=\min \left\{\varepsilon_{3}, \varepsilon_{4}\right\}$. Then, (55) is achieved by (56), (59), and (60). The closeness proof is complete.

\section{Simulation}

In the simulation, we will use two examples to demonstrate the result in our paper. Example 1 employs the model of a DC motor with input and output delays while Example 2 employs a nonlinear model with state delay.

\subsection{Example 1}

Consider a model of a field-controlled DC motor (see Example 4.1 in Bekiaris-Liberis \& Kristic, 2013 and Exercise 1.17 in Khalil, 2002):

$$
\begin{aligned}
& \dot{x}_{1}(t)=-x_{1}(t)+u(t-\tau) \\
& \dot{x}_{2}(t)=-x_{2}(t)-x_{1}(t) x_{3}(t)+1 \\
& \dot{x}_{3}(t)=x_{1}(t) x_{2}(t)-x_{3}(t) \\
& y(t)=x_{3}(t-s)
\end{aligned}
$$

where $x_{1}, x_{2}, x_{3}, u$ are the field current, armature current, angular velocity, and filed voltage, respectively, $\tau$ and $s$ are the input and output delays. We set the equilibrium point on $(0,1,0)$ and the initial conditions are $\left[x_{1}(0), x_{2}(0), x_{3}(0)\right]^{\mathrm{T}}=[0.1,1,0.1]^{\mathrm{T}}$. For the purpose of exhibiting the robustness of high-gain observer with respect to shaft damping, we ignore the damping term $-x_{3}(t)$ in the third equation of (61). Then we take $\eta=x_{2}-1$, $\xi_{1}=x_{3}$, and $\xi_{2}=x_{1} x_{2}$ to transform the model in the following normal form:

$$
\begin{aligned}
& \dot{\eta}(t)=-\eta(t)-\frac{\xi_{1}(t) \xi_{2}(t)}{\eta(t)+1} \\
& \dot{\xi}_{1}(t)=\xi_{2}(t) \\
& \dot{\xi}_{2}(t)=-2 \xi_{2}(t)+\frac{\xi_{2}(t)}{\eta(t)+1}-\frac{\xi_{1}(t) \xi_{2}^{2}(t)}{[\eta(t)+1]^{2}}+[\eta(t)+1] u(t-\tau) \\
& y(t)=\xi_{1}(t-s)
\end{aligned}
$$

We design a state feedback sliding mode control

$$
u(t)=-\mu_{1} \operatorname{sat}\left[\frac{\xi_{1}(t+\tau)+\xi_{2}(t+\tau)}{\mu}\right]
$$

over the compact set $\Omega_{c}=\{|\eta| \leq \hat{c}\} \times\left\{\left|\xi_{1}\right| \leq c\right\} \times\{|\Gamma| \leq c\}$ for some $c$ and $\hat{c}$, where $\mu_{1}$ is a positive constant and $\Gamma=\xi_{1}+\xi_{2}=0$ is the sliding manifold. Under the state feedback control (62), with sufficiently small $\mu$, the origin of the closed-loop system will be exponentially stable. Thus we take $c=0.35, \hat{c}=0.5$, and $\mu_{1}=3.4$, where the set $\Omega_{c}$ includes the initial condition. It shows that exponential stability can be achieved with any $\mu<0.35$. Simulation is performed with $\mu=0.1$.

The high-gain predictor is given by

$$
\begin{aligned}
\dot{\hat{\xi}}_{1}(t)= & \hat{\xi}_{2}(t)+\frac{a_{1}}{\varepsilon}\left[y(t)-\hat{\xi}_{1}(t-s-\tau)\right] \\
\dot{\hat{\xi}}_{2}(t)=- & \hat{\xi}_{2}(t)-\left(4 c^{3}+\mu_{2}\right) \operatorname{sat}\left[\frac{\hat{\xi}_{1}(t) \hat{\xi}_{2}^{2}(t)}{4 c^{3}+\mu_{2}}\right]+u(t) \\
& +\frac{a_{2}}{\varepsilon^{2}}\left[y(t)-\hat{\xi}_{1}(t-s-\tau)\right]
\end{aligned}
$$

with $\hat{\xi}_{1}(0)=\hat{\xi}_{2}(0)=0$, where $\mu_{2}$ is a positive constant. Set the time-delays as $\tau=0.09 \mathrm{~s}, s=0.01 \mathrm{~s}$. The output feedback control is taken as

$$
u(t)=-3.4 \mathrm{sat}\left[\frac{\hat{\xi}_{1}(t)+\hat{\xi}_{2}(t)}{0.1}\right]
$$

We apply the output feedback control to the system (61) to verify that the performance under bounded output feedback control (BOFC) recovers the performance under bounded state feedback control (BSFC) as $\varepsilon$ decreases, as well as its robustness to shaft damping. Taking $\varepsilon=0.9,0.6$, the state trajectories and control inputs are shown in Figure 1. As displayed in Fig. 1, as $\varepsilon$ decreases, the trajectories under output feedback behave much closer to those under state feedback.
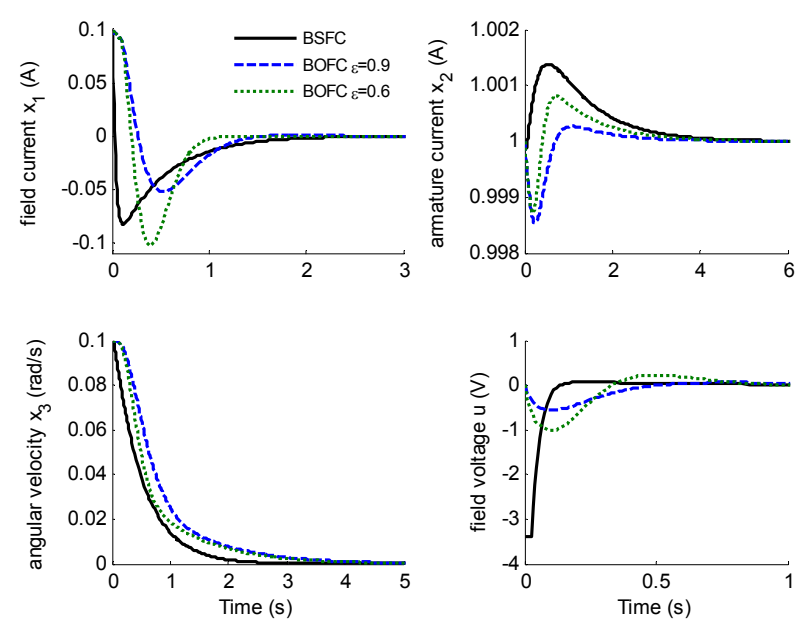

Fig. 1. States and control variables under BSFC and BOFC

Furthermore, recall that our analysis requires a lower bound on $\varepsilon$ of the form $\varepsilon \geq \sigma r$. In order to demonstrate this fact, we fix the delays at $\tau=0.09 \mathrm{~s}, s=0.01 \mathrm{~s}$, and reduce $\varepsilon$ from $0.3,0.2$, to 0.1 . As shown in Fig. 2, as $\varepsilon$ reduces, 
the trajectories begin to oscillate and the performance deteriorates.
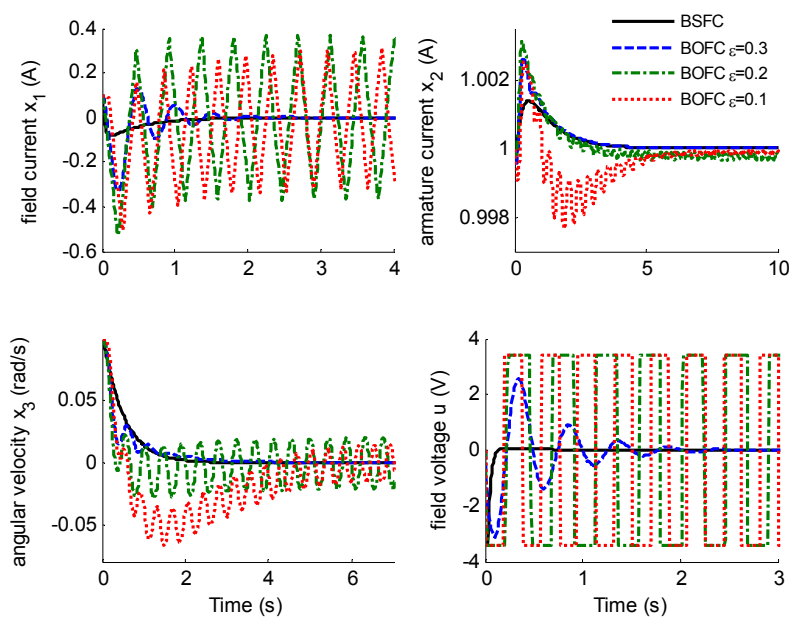

Fig. 2. States and control variables under BSFC and BOFC with different $\varepsilon$

\subsection{Example 2}

Consider the nonlinear system

$$
\begin{aligned}
& \dot{\eta}_{1}(t)=-\eta_{1}(t)+\xi_{1}(t) \\
& \dot{\xi}_{1}(t)=\xi_{2}(t) \\
& \dot{\xi}_{2}(t)=\sin \left(\xi_{1}(t)\right)+\xi_{2}^{2}(t-l)+u(t)+\eta_{1}(t) \\
& y(t)=\xi_{1}(t)
\end{aligned}
$$

where $l$ is the state delay. The initial states are $\left[\eta_{1}(0), \xi_{1}(0), \xi_{2}(0)\right]^{\mathrm{T}}=[0,0.1,0]^{\mathrm{T}}$. Design a state feedback sliding mode control

$u(t)=-\left(4 c^{2}+\mu_{2}\right)$ sat $\left[\frac{\xi_{2}^{2}(t-l)}{4 c^{2}+\mu_{2}}\right]-\left(3 c+1+\mu_{1}\right) \operatorname{sat}\left[\frac{\xi_{1}(t)+\xi_{2}(t)}{\mu}\right]$ over the compact set $\left\{\left|\eta_{1}\right| \leq c\right\} \times\left\{\left|\xi_{1}\right| \leq c\right\} \times\{|\Gamma| \leq c\}$ for some $c$, where $\mu_{1}, \mu_{2}$ are positive constants. Then, with sufficiently small $\mu$, the closed-loop system will be exponentially stable. Design the high-gain predictor:

$$
\begin{aligned}
\dot{\xi}_{1}(t)= & \hat{\xi}_{2}(t)+\frac{a_{1}}{\varepsilon}\left[y(t)-\hat{\xi}_{1}(t)\right] \\
\dot{\hat{\xi}}_{2}(t)= & \sin \left(\hat{\xi}_{1}(t)\right)+\left(4 c^{2}+\mu_{2}\right) \operatorname{sat}\left[\frac{\hat{\xi}_{2}^{2}(t-l)}{4 c^{2}+\mu_{2}}\right]+u(t) \\
& +\frac{a_{2}}{\varepsilon^{2}}\left[y(t)-\hat{\xi}_{1}(t)\right]
\end{aligned}
$$

with $\hat{\xi}_{1}(0)=\hat{\xi}_{2}(0)=0$. We work with $l=0.003, \mu_{1}=\mu_{2}=1$, and $c=0.2$. It can be shown that exponential stability is achieved with any $\mu<0.2$. So simulate with $\mu=0.1$. The output feedback control is

$$
u(t)=-1.16 \text { sat }\left[\frac{\hat{\xi}_{2}^{2}(t-l)}{1.16}\right]-2.6 \mathrm{sat}\left[\frac{\hat{\xi}_{1}(t)+\hat{\xi}_{2}(t)}{0.1}\right]
$$

Taking $\varepsilon=0.1,0.01$, the state trajectories and control inputs are depicted in Fig. 3.
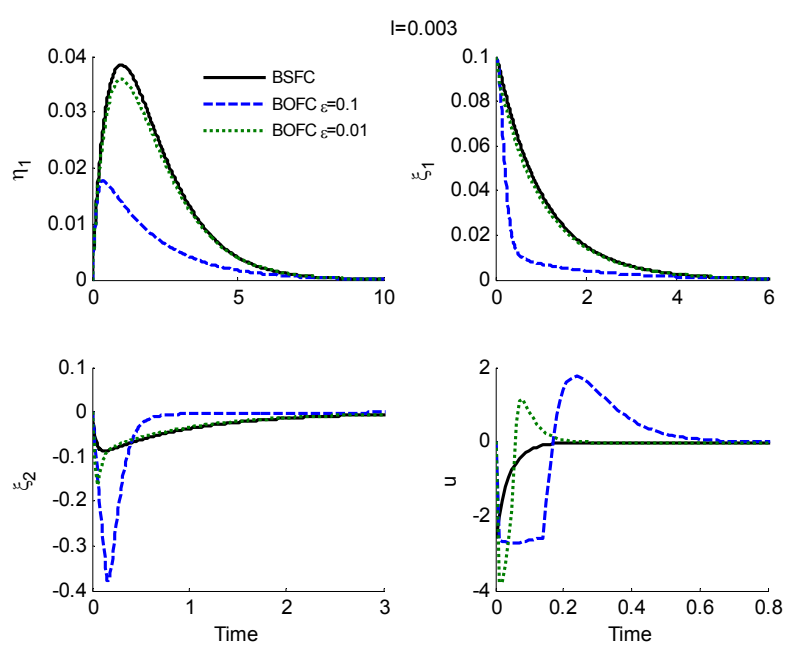

Fig. 3. States and control inputs under BSFC and BOFC

Fig. 3 demonstrates that the performance of the closedloop system under bounded output feedback recovers that under bounded state feedback. As $\varepsilon$ decreases, the trajectories under output feedback get much closer to those under state feedback, which is a manifestation of the performance recovery property of high-gain observers when equipped with a globally bounded control. This implies that the bounded output feedback control works successfully on performance recovery of the closed-loop system. It can be seen the trajectories under BSFC are exponentially stable, where the state delay has been taken into consideration, so that there is no restriction on state delay.

\section{Conclusion}

The paper uses a novel way to study the output feedback control for time-delay nonlinear systems. Using the high-gain predictor, estimates of the predictive state, delayed state, and current-time state are implemented in output feedback. The performance recovery proof demonstrates the advantage of the high-gain predictor-based bounded controller. Meanwhile, analysis requires upper and lower bounds on the observer parameter $\varepsilon$, where the lower bound depends on the input and output time delays. Finally, the effectiveness and feasibility of the proposed controller are illustrated by simulation.

\section{Acknowledgements}

This work was supported in part by the National Science Foundation (U.S.) (ECCS-1128476) and by the National Natural Science Foundation of China (61364012).

\section{References}

Ahmed-Ali, T., Van Assche V., Massieu, J-F., \& Dorleans P. (2013). Continuous-discrete observer for state affine systems with sampled and delayed measurements. IEEE Transactions on Automatic Control, 58(4), 1085-1091. 
Ahmed-Ali, T., Cherrier, E., \& Lamnabhi-Lagarrigue, F. (2012). Cascade high gain predictors for a class of nonlinear systems. IEEE Transactions on Automatic Control, 57(1), 224-229.

Ahmed-Ali, T., Cherrier, E., \& M'Saad, M. (2009). Cascade high gain observers for nonlinear systems with delayed output measurement. $48^{\text {th }}$ IEEE Conference on Decision and Control, Shanghai, China (pp. 8226-8231).

Bekiaris-Liberis, N., \& Kristic, M. (2013). Robustness of nonlinear predictor feedback laws to time- and state-dependent delay perturbations. Automatica, 49(6), 1576-1590.

Farza, M., Sboui, A., Cherrier, E., \& M'Saad, M. (2010). Highgain observer for a class of time-delay nonlinear systems. International Journal of Control, 83(2), 273-280.

Ghanes, M., Leon, J.D., \& Barbot, J.-P. (2013). Observer design for nonlinear systems under unknown time-varying delays. IEEE Transactions on Automatic Control, 58(6), 1529-1534.

Germani, A., Manes, C., \& Pepe, P. (2002). A new approach to state observation of nonlinear systems with delayed output. IEEE Transactions on Automatic Control, 47(1), 96-101.

Germani, A., Manes, C., \& Pepe, P. (2012). Observer-based stabilizing control for a class of nonlinear retarded systems, R. Sipahi, T. Vyhlidal, S.-I. Niculescu, P. Pepe, (EDs), Time Delay Systems Methods, Applications and New Trends, Lecture Notes in Control and Information Science, Springer, 423, 331-342.

Germani, A., \& Pepe, P. (2005). A State observer for a class of nonlinear systems with multiple discrete and distributed time delays. European Journal of Control, 11(3), 196-205.

Kazantzi, N., \&Wright, R. (2005). Nonlinear observer design in the presence of delayed output measurements. Systems \& Control Letters, 54(9), 877-886.

Karafyllis, I., Krstic, M., Ahmed-Ali, T., \& Lamnabhi-Lagarrigue, F. (2014). Global stabilization of nonlinear delay systems with a compact absorbing set. International Journal of Control, 87(5), 1010-1027.

Karafyllis, I. (2006). Lyapunov theorems for systems described by retarded functional differential equations. Nonlinear Analysis, 64(3), 590-617.

Khalil, H.K. (2002). Nonlinear Systems, 3rd Edition. New York: Prentice-Hall, Upper Saddle River.

Kharitonov, V.L. (2012). Time-Delay Systems: Lyapunov Functions and Matrices. Birkhäuser .

Krasovskii, N.N. (1963). Stability of Motion. California: Stanford University Press.

Lei, J., \& Khalil, H.K. (2016). Feedback linearization for nonlinear systems with time-varying input and output delays by using high-gain predictor. IEEE Transactions on Automatic Control, 61(8), 2016, (published online in 2015).

Luse, D.W., \& Khalil, H.K. (1985). Frequency domain results for systems with slow and fast dynamics. IEEE Transactions on Automatic Control, AC-30(12), 1171-1179.

\section{Appendix A. Proof for Lemma 1}

Lemma 1. Consider time-delay system

$$
\varepsilon \dot{x}(t)=f\left(x_{t}\right)
$$

where $f: \mathcal{D} \rightarrow R^{\bar{n}}$ is continuous and Lipschitz with $f(0)=0$, and $\mathcal{D}=\left\{x \in R^{\bar{n}} \mid\left\|x_{t}\right\|_{\mathrm{s}}<b_{1}\right\}$. Let $v, \phi, b_{0}$ be positive constants with $b_{0}<b_{1} / v$, and $\mathcal{D}_{0}=\left\{x \in R^{\bar{n}}\left\|x_{t_{0}}\right\|_{s}<b_{0}\right\}$. Assume the solution $x\left(t ; t_{0}, \varphi\right)$ of the system (63) satisfies

$$
\left\|x\left(t ; t_{0}, \varphi\right)\right\| \leq v\left\|x_{t_{0}}\right\|_{s} \mathrm{e}^{-\frac{\phi}{\varepsilon}\left(t-t_{0}\right)}, \quad \forall x_{t_{0}} \in \mathcal{D}_{0}, \quad \forall t \geq t_{0}
$$

Then, there is a functional $V_{4}\left(x_{t}, t, \varepsilon\right): \mathcal{D}_{0} \times R \times R^{+} \rightarrow R^{+}$that satisfies the inequalities

$$
\begin{aligned}
\bar{c}_{1}\left\|x_{t}\right\|_{s}^{2} \leq V_{4}\left(x_{t}, t, \varepsilon\right) & \leq \bar{c}_{2}\left\|x_{t}\right\|_{s}^{2} \\
\dot{V}_{4}\left(x_{t}, t, \varepsilon\right) & \leq-\frac{\bar{c}_{3}}{\varepsilon}\left\|x_{t}\right\|_{s}^{2} \\
\left|V_{4}\left(x_{t}^{\prime \prime}, t, \varepsilon\right)-V_{2}\left(x_{t}^{\prime}, t, \varepsilon\right)\right| & \leq \bar{c}_{4}\left(\left\|x_{t}^{\prime \prime}\right\|_{s}+\left\|x_{t}^{\prime}\right\|_{s}\right)\left\|x_{t}^{\prime \prime}-x_{t}^{\prime}\right\|_{s}
\end{aligned}
$$

for some positive constants $\bar{c}_{1}, \bar{c}_{2}, \bar{c}_{3}, \bar{c}_{4}$

If the origin is globally exponentially stable and the assumptions hold globally, then $V_{4}$ is defined and satisfies (65-1)-(65-3) for $\mathcal{D}_{0}=R^{\bar{n}}$.

Proof. We follow the proof of Lemma 33.1 of Krasovskii (1963) with a modification of the definition of the Lyapunov functional. Let

$$
\begin{aligned}
V_{4}\left(x_{t}, t, \varepsilon\right)= & \frac{1}{\varepsilon} \int_{t}^{t+\varepsilon \Delta}\|x(\varpi+\theta ; t, x)\|_{s}^{2} \mathrm{~d} \varpi \\
& +\sup _{\varpi \in[t, t+\varepsilon \Delta]}\|x(\varpi+\theta ; t, x)\|_{s}^{2}
\end{aligned}
$$

where $\Delta$ denotes a time interval, which will be determined later on, and $\theta \in[-r, 0]$. The left inequality of (65-1) follows from

$$
V_{4}\left(x_{t}, t, \varepsilon\right) \geq \sup _{\sigma \in[t, t+\varepsilon \Delta]}\left\|x_{\sigma}\right\|_{s}^{2} \geq\left\|x_{t}\right\|_{s}^{2}
$$

where $\bar{c}_{1}=1$. The right inequality of (65-1) follows from (63) and the property

$$
\begin{aligned}
V_{4}\left(x_{t}, t, \varepsilon\right) & \leq \frac{1}{\varepsilon} \int_{t}^{t+\varepsilon \Delta} v^{2}\left\|x_{t}\right\|_{s}^{2} \mathrm{e}^{-\frac{2 \phi}{\varepsilon}(\sigma-t)} \mathrm{d} \varpi+\sup _{\sigma \in[t, t+\varepsilon \Delta]}\left[v^{2}\left\|x_{t}\right\|_{s}^{2} \mathrm{e}^{-\frac{2 \phi}{\varepsilon}(\pi-t)}\right] \\
& \leq-\left.\frac{v^{2}}{2 \phi}\left\|x_{t}\right\|_{s}^{2} \mathrm{e}^{-\frac{2 \phi}{\varepsilon}(\pi-t)}\right|_{t} ^{t+\varepsilon \Delta}+v^{2}\left\|x_{t}\right\|_{s}^{2} \\
& \leq v^{2}\left[1+\frac{1}{2 \phi}\left(\mathrm{e}^{-2 \phi \Delta}-1\right)\right]\left\|x_{t}\right\|_{s}^{2}
\end{aligned}
$$

with

$$
\bar{c}_{2}=v^{2}\left[1+\frac{1}{2 \phi}\left(\mathrm{e}^{-2 \phi \Delta}-1\right)\right]
$$

The inequality (64) shows that the second term of the right member of the functional (66) does not increase with increasing $t$ along the trajectories of the system (63). Thus, 


$$
\begin{aligned}
& \dot{V}_{4}=\lim _{h \rightarrow 0^{+}} \sup \frac{1}{h}\left[V_{4}\left(x_{t+h}, t+h, \varepsilon\right)-V_{4}\left(x_{t}, t, \varepsilon\right)\right] \\
& =\lim _{h \rightarrow 0^{+}} \sup \frac{1}{h}\left[\frac{1}{\varepsilon}\left(\int_{t+h}^{t+h+\varepsilon s}\left\|x_{\sigma}\right\|_{s}^{2} \mathrm{~d} \pi-\int_{t}^{t+\varepsilon s}\left\|x_{\sigma}\right\|_{S}^{2} \mathrm{~d} \varpi\right)\right. \\
& \left.+\left(\sup _{\varpi \in[t+h, t+h+\varepsilon \Delta]}\left\|x_{\varpi}\right\|_{s}^{2}-\sup _{\sigma \in[t, t+\varepsilon \Delta]}\left\|x_{\varpi}\right\|_{s}^{2}\right)\right] \\
& \leq \lim _{h \rightarrow 0^{+}} \sup \frac{1}{h \varepsilon}\left(\int_{t+h}^{t+h+\varepsilon \Delta}\left\|x_{\varpi}\right\|_{s}^{2} \mathrm{~d} \varpi-\int_{t}^{t+\varepsilon \Delta}\left\|x_{\varpi}\right\|_{s}^{2} \mathrm{~d} \varpi\right) \\
& \leq \lim _{h \rightarrow 0^{+}} \sup \frac{1}{h \mathcal{E}}\left(\int_{t+\varepsilon \Delta}^{t+h+\varepsilon \Delta}\left\|x_{\sigma}\right\|_{s}^{2} \mathrm{~d} \sigma-\int_{t}^{t+h}\left\|x_{\sigma}\right\|_{s}^{2} \mathrm{~d} \sigma\right) \\
& =\frac{1}{\varepsilon}\left(\left\|x_{t+\varepsilon \Delta}\right\|_{s}^{2}-\left\|x_{t}\right\|_{s}^{2}\right)
\end{aligned}
$$

Due to (64), (67) becomes

$$
\begin{aligned}
\dot{V}_{4} & \leq \frac{1}{\varepsilon}\left(v^{2}\left\|x_{t}\right\|_{s}^{2} \mathrm{e}^{-2 \phi \Delta}-\left\|x_{t}\right\|_{s}^{2}\right) \\
& =\frac{1}{\varepsilon}\left(v^{2} \mathrm{e}^{-2 \phi \Delta}-1\right)\left\|x_{t}\right\|_{s}^{2} \\
& \leq-\frac{1}{2 \varepsilon}\left\|x_{t}\right\|_{s}^{2}-\frac{1}{2 \varepsilon}\left(1-2 v^{2} \mathrm{e}^{-2 \phi \Lambda}\right)\left\|x_{t}\right\|_{s}^{2}
\end{aligned}
$$

Choosing $\Delta \geq \frac{1}{2 \phi} \ln \left(2 v^{2}\right)$ ensures the second term in the right-hand side of the inequality (68) is negative, which indicates (65-2) is true with $\bar{c}_{3}=1 / 2$. Furthermore,

$$
\begin{aligned}
& \left|V_{4}\left(x_{t}^{\prime \prime}, t, \varepsilon\right)-V_{2}\left(x_{t}^{\prime}, t, \varepsilon\right)\right| \\
& \leq \frac{1}{\varepsilon} \int_{t}^{t+\varepsilon \Lambda}\left[\left\|x\left(\varpi+\theta ; t, x_{t}^{\prime \prime}\right)\right\|_{s}^{2}-\left\|x\left(\varpi+\theta ; t, x_{t}^{\prime}\right)\right\|_{s}^{2}\right] \mathrm{d} \varpi \\
& +\sup _{\varpi \in[t, t+\varepsilon \varepsilon]}\left\|x\left(\varpi+\theta ; t, x_{t}^{\prime \prime}\right)\right\|_{s}^{2}-\sup _{\sigma \in[t, t+\varepsilon \varepsilon]}\left\|x\left(\varpi+\theta ; t, x_{t}^{\prime}\right)\right\|_{s}^{2} \\
& \leq \frac{1}{\varepsilon} \int_{t}^{t+\varepsilon \Delta}\left(\left\|x\left(\varpi+\theta ; t, x_{t}^{\prime \prime}\right)\right\|_{s}+\left\|x\left(\varpi+\theta ; t, x_{t}^{\prime}\right)\right\|_{s}\right) \\
& \times\left|\left(\left\|x\left(\varpi+\theta ; t, x_{t}^{\prime \prime}\right)\right\|_{s}-\left\|x\left(\varpi+\theta ; t, x_{t}^{\prime}\right)\right\|_{s}\right)\right| \mathrm{d} \varpi \\
& +\sup _{\varpi \in[t, t+\varepsilon \varepsilon]}\left(\left\|x\left(\varpi+\theta ; t, x_{t}^{\prime \prime}\right)\right\|_{s}+\left\|x\left(\varpi+\theta ; t, x_{t}^{\prime}\right)\right\|_{s}\right) \\
& \times\left|\left(\left\|x\left(\varpi+\theta ; t, x_{t}^{\prime \prime}\right)\right\|_{s}-\left\|x\left(\varpi+\theta ; t, x_{t}^{\prime}\right)\right\|_{s}\right)\right| \\
& \leq \frac{1}{\varepsilon} \int_{t}^{t+\varepsilon \Delta}\left(\left\|x\left(\varpi+\theta ; t, x_{t}^{\prime \prime}\right)\right\|_{s}+\left\|x\left(\varpi+\theta ; t, x_{t}^{\prime}\right)\right\|_{s}\right) \\
& \times\left\|x\left(\varpi+\theta ; t, x_{t}^{\prime \prime}\right)-x\left(\varpi+\theta ; t, x_{t}^{\prime}\right)\right\|_{s} \mathrm{~d} \varpi \\
& +\sup _{\varpi \in[t, t+\varepsilon)]}\left(\left\|x\left(\varpi+\theta ; t, x_{t}^{\prime \prime}\right)\right\|_{s}+\left\|x\left(\sigma+\theta ; t, x_{t}^{\prime}\right)\right\|_{s}\right) \\
& \times\left\|x\left(\varpi+\theta ; t, x_{t}^{\prime \prime}\right)-x\left(\varpi+\theta ; t, x_{t}^{\prime}\right)\right\|_{s}
\end{aligned}
$$

According to Lemma 27.1 (p. 128, Krasovskii, 1963),

$$
\left\|x\left(\varpi+\theta ; t, x_{t}^{\prime \prime}\right)-x\left(\varpi+\theta ; t, x_{t}^{\prime}\right)\right\|_{s} \leq\left\|x_{t}^{\prime \prime}-x_{t}^{\prime}\right\|_{s} \mathrm{e}^{\frac{\bar{L}}{\varepsilon}(\sigma-t)}
$$

where $\bar{L}$ is the Lipschitz constant of $f$. Due to (64) and (70), (69) becomes

$$
\begin{aligned}
& \left|V_{4}\left(x_{t}^{\prime \prime}, t, \varepsilon\right)-V_{2}\left(x_{t}^{\prime}, t, \varepsilon\right)\right| \\
& \leq \frac{1}{\varepsilon} \int_{t}^{t+\varepsilon \Delta}\left(\left\|x\left(\varpi+\theta ; t, x_{t}^{\prime \prime}\right)\right\|_{s}+\left\|x\left(\varpi+\theta ; t, x_{t}^{\prime}\right)\right\|_{s}\right) \\
& \times\left\|x\left(\varpi+\theta ; t, x_{t}^{\prime \prime}\right)-x\left(\varpi+\theta ; t, x_{t}^{\prime}\right)\right\|_{s} \mathrm{~d} \varpi \\
& +\sup _{\varpi \in[t, t+\varepsilon \varepsilon]}\left(\left\|x\left(\varpi+\theta ; t, x_{t}^{\prime \prime}\right)\right\|_{s}+\left\|x\left(\varpi+\theta ; t, x_{t}^{\prime}\right)\right\|_{s}\right) \\
& \times\left\|x\left(\varpi+\theta ; t, x_{t}^{\prime \prime}\right)-x\left(\varpi+\theta ; t, x_{t}^{\prime}\right)\right\|_{s} \\
& \leq \frac{1}{\varepsilon} \int_{t}^{t+\varepsilon \Delta} \mathrm{e}^{\frac{\bar{L}-\phi}{\varepsilon}(\sigma-t)} \mathrm{d} \sigma\left(\left\|x_{t}^{\prime \prime}\right\|_{s}+\left\|x_{t}^{\prime}\right\|_{s}\right)\left\|x_{t}^{\prime \prime}-x_{t}^{\prime}\right\|_{s} \\
& +v \mathrm{e}^{(\bar{L}-\phi) \Delta}\left(\left\|x_{t}^{\prime \prime}\right\|_{s}+\left\|x_{t}^{\prime}\right\|_{s}\right)\left\|x_{t}^{\prime \prime}-x_{t}^{\prime}\right\|_{s} \\
& \leq\left[\frac{1}{\bar{L}-\phi}\left(\mathrm{e}^{(\bar{L}-\phi) \Delta}-1\right)+v \mathrm{e}^{(\bar{L}-\phi) \Delta}\right]\left(\left\|x_{t}^{\prime \prime}\right\|_{s}+\left\|x_{t}^{\prime}\right\|_{s}\right)\left\|x_{t}^{\prime \prime}-x_{t}^{\prime}\right\|_{s}
\end{aligned}
$$

which implies (65-3) is true with

$$
\bar{c}_{4}=\frac{1}{\bar{L}-\phi}\left(\mathrm{e}^{(\bar{L}-\phi) \Delta}-1\right)+v \mathrm{e}^{(\bar{L}-\phi) \Delta}
$$

If the assumptions hold globally, then $b_{0}$ can be chosen arbitrarily large.

The Lemma 1 is proved. 